University of San Diego

Digital USD

Spring 5-25-2019

\title{
Remifentanil Patient Controlled Analgesia Use in Laboring Women: A Feasibility Pilot Project
}

Joshua Aaron Carr

University of San Diego, joshuacarr@sandiego.edu

Follow this and additional works at: https://digital.sandiego.edu/dnp

Part of the Nursing Commons

\section{Digital USD Citation}

Carr, Joshua Aaron, "Remifentanil Patient Controlled Analgesia Use in Laboring Women: A Feasibility Pilot Project" (2019). Doctor of Nursing Practice Final Manuscripts. 86.

https://digital.sandiego.edu/dnp/86

This Doctor of Nursing Practice Final Manuscript is brought to you for free and open access by the Theses and Dissertations at Digital USD. It has been accepted for inclusion in Doctor of Nursing Practice Final Manuscripts by an authorized administrator of Digital USD. For more information, please contact digital@sandiego.edu. 
Running Head: REMIFENTANIL IN LABOR

Remifentanil Patient Controlled Analgesia Use in Laboring Women: A Feasibility Pilot Project

Joshua A. Carr DNP, CRNA

Joseph Burkard, DNSc, CRNA

Alia Shbeeb, MD, FACOG

University of San Diego

Author Note

University of San Diego, Hahn School of Nursing \& Health Science

5998 Alcala Park, San Diego, CA 92110 


\section{Introduction of Problem}

Silva and Halpern (2010) note that pain during childbirth is widely recognized as one of the most distressing events that women can experience. The anticipation or experience of labor pain might be even more troubling for those women who do not have the option of Epidural Analgesia (EA) for whatever reason. The desire to ameliorate this pain experience has resulted in exploration into alternative methods for addressing labor pain. A growing volume of research has pointed to remifentanil as a promising medication, due to its fast onset and short half-life (Blair, Dobson, Hill, McCracken, \& Fee, 2005; Ohashi, Baghirzada, Sumikura, \& Balki, 2016; Weibel et. al, 2017). Evidence as to the superiority of patient controlled analgesia (PCA) over other modalities is also mounting (Lee et al., 2017). Consequently, the acceptance of PCA therapy in labor is spreading, especially outside the United States (Devabhakthuni, 2013).

Despite findings that point to a high safety profile and efficacious analgesic and satisfaction properties, a recent Cochrane review indicated that there is not enough high-quality evidence to make a strong recommendation of support before larger trials are conducted (Weibel et. al., 2017). Other systematic reviews, however, have found enough evidence to make recommendations for implementing remifentanil PCA into clinical practice, particularly where EA is not a viable option due to geographic limitations, comorbidities, or cultural/personal preferences (Devabhakthuni, 2013; Ohashi et. al., 2016; Velde \& Carvalho, 2015). In order to expand the body of evidence around remifentanil PCA (RPCA) and optimize dosing regimens future research should abandon the attempt to prove superiority to EA; rather, the next wave of studies should adopt RPCA as the current best practice method in patients not able to receive EA (Douma, Verwey, Kam-Endtz, van der Linden \& Stienstra, 2015; Tamagawa \& Weaver, 2012). 
This paper will detail the initial phase of a pilot project focused on implementing a RPCA protocol in a 15 bed labor and delivery ward.

\section{Background \& Significance}

Epidural analgesia for the management of labor pain has increased in popularity over the past 4 decades. The current prevalence of this technique in the United States is approximately 60\% (Silva \& Halpern, 2010; Meng \& Smiley, 2017). However, many women are unable to receive an epidural during labor due to contraindications, such as coagulopathy, infection, or spinal irregularity. Some parturients also elect alternative pain management strategies, such as systemic opioids, for personal or cultural reasons (Tveit, Seiler, Halvorsen, \& Rosland, 2012). Current alternative techniques (AT) for pain management in labor without EA vary from intermittent administration of opioids or other analgesics to hypnosis. The most promising alternative strategy is RPCA. An early example of research in this area by Evron, Glezerman, Sadan, Boaz, and Ezri (2005) demonstrated significant improvement in both pain (35.8 compared to 58.8 on a visual analog scale) and satisfaction (3.9 versus 1.9 satisfaction score) when they examined RPCA against meperidine IV infusion. In fact, one meta-analysis looking at randomized controlled trials found that there was no significant difference on measures of maternal satisfaction between those receiving EA and those controlling a RPCA delivery system (OR 1.3, CI 0.31-5.38) (Lee et al., 2017). RPCA involves the self-administration of pain medication by the patient through the use of a demand button connected to an infusion pump. Recent systematic reviews and meta-analysis confirm that remifentanil demonstrates better pain control and offers safety advantages when compared to other intravenous medications (Ohashi et. al., 2016; Weibel et al., 2017). Remifentanil PCA is an evidence based intervention for labor pain that should be offered to women who cannot receive EA. 


\section{Literature Review}

The primary strength of the evidence related to utilization of remifentanil is the continued demonstration of superiority to other opioid medications on key variables. In one relatively small study, Ng et al. (2011) using found that remifentanil provided significantly better pain reduction, duration of pain control, and maternal satisfaction without associated side effects. Ohashi et al. (2016) amplified these findings in their systematic review and concluded that "remifentanil appears to have a significant role in pain relief during labor" (p.1026). None of the studies reviewed, including a recent meta-analysis, demonstrated equivocation about the efficacy of remifentanil when compared to other opioid medications studied (Weibel, et al., 2017).

PCA as a technique was identified as a possible independent contributor to increased satisfaction among the participants as it may reinforce self-efficacy as a factor in satisfaction (Ng, et al., 2011). In their investigation, $\mathrm{Ng}$ and colleagues (2011) utilized doses of 25 or $30 \mathrm{mcg}$ of remifentanil available roughly every four minutes for self-administration as compared to a placebo PCA coupled with meperidine IM injection and found that RPCA not only reduced relative pain scores by $44 \%$ at 2 hours, but also had significantly better overall satisfaction ( 8 versus 6 on a measurement scale). Notably, even when matched against other medications using PCA delivery, remifentanil showed better pain relief (Blair et. al., 2005; Douma, et al., 2010). Many researchers have compared remifentanil to EA on pain relief measures where it often falls short of matching the epidural group (Anim-Somuah, et al., 2011; Douma, et al., 2015; Storac, et al., 2015). Despite this potentially unfavorable comparison, other researchers found that remifentanil performed nearly as well as EA on both pain and satisfaction measures, particularly early in labor (Douma, et al., 2010; Tveit, Seiler, Halvorsen, \& Rosland, 2012; Frauenfelder et. al., 2015). 
According to Weibel et al. (2017), the evidence surrounding remifentanil PCA as it pertains to parturients is hampered by a dearth of high-quality studies. This lack of a large multicenter investigation is probably the largest weakness in the evidence base supporting remifentanil PCA. Some studies have shown that the effectiveness of RPCA may be short-lived, resulting in diminishing pain relief through labor progression and requiring rescue intervention (Douma, et al., 2010). Further exploration in this area will surely occur, but other challenges to the widespread implementation of RPCA will remain. Even if evidence of the effectiveness of this technique continues to accumulate, caregiver resistance is likely to remain a serious obstacle (Ohashi et. al., 2016). Many investigators have implicated remifentanil as higher risk medication due to episodes of respiratory depression and sedation. However, there is little if any evidence of poor outcomes associated with these episodes (Weibel, et al., 2017). Fear of harmful effects that necessitate additional personnel for one-to-one care and requirements for extra equipment such as end-tidal carbon dioxide (ETCO2) monitoring could impede timely progress in collecting the breadth of data required to support widespread acceptance of remifentanil PCA on the labor ward.

\section{Design}

This is a retrospective, data only, pilot feasibility project examining the process of implementing a new evidence based pain control technique for labor. The purpose of this project is to reduce the pain experience of women going through childbirth who are unable to receive a continuous lumbar epidural infusion due to contraindication. Baseline data was culled from a records review of all live births at one medium sized health system hospital over a 3 month period. Patients with a live birth during this period who labored without EA were identified by a database search. After that group was identified, mean pain scores were calculated using all 
recorded pain scores collected after the first report of pain during admission to the labor ward and continuing through delivery. Comparisons were made based upon the identified reason for not receiving EA. Reasons for not receiving EA included medical conditions (e.g. drug therapy, thrombocytopenia, or spinal abnormality), elective unmedicated labors, elective intravenous or intramuscular opioids as a primary pain management plan, and inability to receive an epidural due to timing (i.e. late request or imminent delivery). The identified reasons formed the basis for creating the following subcategories: (a) medical condition, (b) natural, (c) opioid plan, and (d) timing. Refer to Table 1 for a distribution of the subcategories. Patients delivering via caesarian section were excluded from pain score average, but were counted to estimate a baseline percentage rate of delivery type. After implementation of the RPCA protocol, pain scores will be extracted from the electronic health record (EHR) for each RPCA recipient and averaged for the entire course of administration. Average pain scores will then be compared between the two groups. Beyond mean pain score, variables identified for examination are rates of caesarian section, labor time, and 5 minute APGAR score. Baseline values for these complications are not detailed here because they will be determined after the implementation of the RPCA protocol during the second phase of the pilot program. Two critical adverse events, unplanned admission of the neonate, and apnea requiring administration of naloxone in either the mother or the neonate, will be identified for the purpose of determining baseline rates.

\section{Results Phase One}

All live births from the labor and delivery ward at a medium sized community hospital over a three-month period were reviewed for inclusion in the preliminary data gathering phase of this pilot project. In all, there were 769 live births. Of that total, 235 occurred by caesarian section resulting in an overall 30.6\% surgical delivery rate. The remaining 534 births consisted 
of 521 normal spontaneous vaginal deliveries (NSVDs), a number that includes 15 vaginal births after caesarian (VBACs) (2.8\%), and 13 forceps or vacuum assisted deliveries $(2.4 \%)$.

The population of interest was identified by excluding parturients delivering by caesarian section as well as those that received a continuous labor epidural regardless of delivery method. The remaining subjects number a total of 140 women, 11 of these were excluded from pain score calculations due to an absence of any recorded pain score in the medical record. Among the patients that delivered vaginally without EA, the distribution of subcategories was as follows: (a) medical condition $(n=8)$ accounted for $5.7 \%$ of the group, (b) natural $(n=54)$ made up $38.6 \%$, (c) opioid plan $(n=19) 13.6 \%$, while (d) timing $(n=59)$ was the most common reason at $42.1 \%$.

The average pain score for the complete group $(n=129)$ was 6.9 on a standard selfreported 0-10 numerical scale. The average pain score of those that received IV or IM opioids (including 11 participants that planned for an unmedicated delivery but elected to receive opioids during the course of labor) $(n=44)$ was $6.0 / 10$, compared to those who did not receive opioids $(n=85)$ whose mean reported pain score was 7.4/10. This a difference between groups of 1.4. These results, shown in Table 2, demonstrate a significant reduction of reported pain scores among the group receiving opioids $(\mathrm{p}=0.0002)$.

There was a total of six different opioid therapies utilized during the review period these were: (a) butorphanol $2 \mathrm{mg}$ IV given once $(\mathrm{n}=12)$, (b) fentanyl 50-100 mcg given up to three times ( $\mathrm{n}=14)$, (c) nalbuphine $10 \mathrm{mg}$ IV given once $(\mathrm{n}=4)$, (d) butorphanol $2 \mathrm{mg}$ IV and fentanyl $50 \mathrm{mcg}$ IV each given once ( $\mathrm{n}=2$ ), (e) nalbuphine $10 \mathrm{mg}$ IM given once, and (f) morphine $10 \mathrm{mg}$ IM given once. The average pain score of the group receiving only butorphanol $2 \mathrm{mg}$ IV (group a) was 5.4/10. The IV fentanyl only group had a mean pain score of 6.9/10. This demonstrates a 
significant difference of 1.5 between these two most frequently used opioid techniques $(\mathrm{p}=0.0227)$.

The target group for this pilot project are those parturients that are denied EA due to a medical condition. This group $(n=9)$ accounted for $1.2 \%$ of women having a live birth over this 3 month period. Of these nine women, one delivered by caesarian section and required planned general anesthesia (GA). The remaining eight participants delivered vaginally. Seven out of eight $(87.5 \%)$ of these women elected to receive some type of opioid for the management of their labor pain. No single technique demonstrated a majority within this subgroup (butorphanol only $n=2$, fentanyl only $n=2$, nalbuphine only $n=2$, butorphanol and fentanyl $n=1$ ). This group reported a mean pain score of 5.5 which is similar to the general opioid group score reported earlier.

\section{Discussion}

Overall, opioid medications offered a significant reduction in pain scores for laboring women. Women who had a medical condition precluding them from receiving EA had a higher rate of opioid use. This characteristic reinforces the appropriate nature of exploring optimal opioid protocols for this population. Drawing conclusions about the best of the identified techniques is problematic due to the likely variation of technique by stage of labor, anticipated time to delivery, and provider specific preferences.

The greatest pain reduction effect was observed with butorphanol, but it is likely that this reflects the longer duration of action (4-6 hours). When choosing butorphanol it is possible that providers anticipated that labor would continue for a longer period of time as compared to the anticipated labor time when fentanyl was selected. Fentanyl has a shorter duration of action (30- 
60 minutes) making it less likely to have lingering effects when delivery is expected in a shorter period of time.

Intermittent IV administration of either butorphanol or fentanyl were the most commonly used techniques in the group receiving IV medications. Butorphanol demonstrated an advantage pain relief advantage over fentanyl; however, the pharmacodynamics of butorphanol may make it an inappropriate selection for women in later stages of labor due to the prolonged somnolence often observed with this medication. In this investigation, intermittent administration of IV fentanyl was inferior to IV butorphanol on reducing the reported pain scale. Fentanyl also failed to impact reported pain when compared to the larger group. Clearly, both techniques fall short of the goal of providing a satisfactory strategy for many patients. Reduction of pain scores, while significant, did not approach the levels reported in the literature for remifentanil.

\section{Intervention - Phase Two}

The purpose of this project is to answer the question: For women experiencing childbirth related pain, what is the effect of remifentanil patient controlled analgesia compared to intermittent intravenous medication administered by a registered nurse? Women experiencing labor pain without EA have an identified need for an opioid based pain relief plan. Pregnant women in labor at Kaiser Permanente Riverside Medical Center (KPRMC) do not have a clear 'best' alternative to EA when requesting pain relief during labor. Current practice is for registered nurses to administer bolus doses of IV pain medicine at variable intervals resulting in uneven and often ineffective pain control. Researchers have found enough evidence to make recommendations for implementing remifentanil PCA into clinical practice, particularly where EA is not a viable option due to geographic limitations, comorbidities, or personal preferences. 
The first phase of this project revealed that the target patient population at this clinical site has an approximate $1.2 \%$ prevalence of parturients who are denied EA due to some comorbidity or anti-coagulant therapy. Extrapolating that figure to an annual rate would indicate that approximately 36 women per year are excluded from EA, the gold standard for pain relief during labor. Data also indicate that this subgroup is more likely to request parenteral opioids than the population of laboring women. Preliminary data indicate that the current best practice at this hospital is administration of butorphanol. Butorphanol administration demonstrated a significant reduction in pain scores in this population; however, the associated somnolence might diminish the feeling of control theorized to play a part in patient satisfaction with pain management (Frauenfelder et. al., 2015). Additionally, butorphanol, like many other intravenous opioids carries the risk of respiratory depression in neonates that requires the administration of naloxone (Anderson, 2011).

Remifentanil is an ultra-short acting pharmaceutical agent that has been widely used in anesthesia for surgery and for the sedation of ventilated patients. It is a mu opioid receptor agonist with a rapid onset and offset that results in profound analgesia for a brief period of time. The context sensitive half-life of remifentanil is only three minutes, a desirable characteristic in labor and delivery due to the overarching concern of respiratory depression in infants due to placental transfer. Metabolism of this drug occurs outside of renal or hepatic pathways via plasma and tissue esterases. These qualities make it suitable for PCA administration in the setting of intermittent intense pain, such as the pain of contraction during labor (Devabhakthuni, 2013).

Multiple randomized controlled trials have shown that remifentanil consistently reduces pain scores or visual analog scales of reported pain by 3 units of measure on a 0 to 10 scale when 
compared to other opioids (Ohashi et. al., 2017). Applying these results to the population of interest at this clinical site, it appears that RPCA has a strong potential to outperform the current practice of intermittent IV medication regardless of technique. The Cochrane review comparing remifentanil to other parenteral methods also demonstrated a standard mean satisfaction score that was 2.11 times higher than the alternative opioid technique (Weibel et. al., 2017). Weibel and colleagues (2017) also examined other variables of interest such as adverse side effects (i.e. pruritis) and serious complications (i.e. non-reassuring fetal status) and found no evidence of increased risk or some benefit to using RPCA as compared to IV or IM opioids.

The use of remifentanil has spread throughout many countries in Europe where it is overtaking meperidine as the drug of choice for labor PCA. In the United Kingdom, remifentanil is the most commonly used agent and PCA use is widespread with nearly $50 \%$ of labor wards offering this strategy for pain management (Ohashi et. al., 2017). Utilization in the United States is much lower. A recent survey by Aaronson and colleagues (2017) revealed that only $31 \%$ of academic medical centers in the United States offer RPCA to laboring women. Those hospitals that did use remifentanil did so infrequently, with no site using it more than 20 times in the previous year (Aaronson et. al., 2017).

\section{Development and Implementation}

In a recent unpublished guideline issued by the pain management committee, this clinical site indicated that improvement of obstetrical pain management is an organizational priority and specifically mentions remifentanil PCA as a strategy for women with contraindications to EA; this communication provides evidence of support for this project (Kaiser Permanente, 2017, October, 30). In 2017, the pain management committee recommended that health system facilities should adopt remifentanil PCA as the current best practice method in patients not able 
to receive EA. A letter of support from the Nursing Director of labor and delivery at the investigation site provides for additional proof of organizational commitment to improving the pain experience of parturients by introducing RPCA.

The Model that will guide this EBP project is the revised Iowa model (Iowa Model Collaborative, 2017). The need for a multidisciplinary team for this project involving medication procurement, ordering, and a relatively high degree of resource allocation makes the Iowa model a strong framework for this project. The matching of new knowledge with a clinical problem creates a strong foundation for a quality improvement project and the support provided by the institutional goal statement allows for the opportunity to deploy this new strategy with the investment of organizational resources.

Staff teaching and stakeholder presentations were conducted in February and March 2018. Institutional Review Board (IRB) approval was obtained from Kaiser Permanente Southern California and the University of San Diego (USD) before data collection began. Phase one data collection began in October 2018 and continued for a period of 3 months. Records review during phase one revealed no episodes of any of the adverse events of interest among the target population. Phase two will commence with the availability of remifentanil, which is currently in shortage, and continue through October 2019. Once available, a dosing order set utilizing a $30 \mathrm{mcg}$ remifentanil bolus only technique coupled with a 2 minute lockout period and allowing for incremental increases to a maximum of $50 \mathrm{mcg}$ will be released for use by the obstetrical staff. During phase two, data will be collected from the first 12 patients enrolled in the RPCA protocol and then compared to baseline figures on the matched variables. Data will be collected from the EHR database to establish the any effect on the following variables in labor and delivery patients enrolled in the RPCA protocol as compared to those in the phase one 
medical condition group. The variables of interest are: (a) pain scores; (b) delivery method; and (c) APGAR scores.

Beyond the three critical adverse events listed earlier (unplanned admission of the neonate, unplanned caesarian section, and apnea requiring administration of naloxone), additional information will be collected on this first cohort of patients receiving the remifentanil protocol including:

- $\quad$ oxygen desaturation events (SPO2<93\%),

- dosage (initial and adjusted),

- change of analgesic method,

- complaint of nausea requiring intervention or vomiting, and

- complaint of pruritus.

Process review will then take place to determine if program restructuring is required. Data collection will then be continued to a total enrollment of 30 patients with restructured guidelines if significant opportunities to improve are identified. After 30 patients complete the remifentanil protocol, outcome measures will be analyzed to determine if expansion or discontinuation of this protocol is indicated. An increase in complication rate of $>5 \%$ will trigger a review of dosing guidelines. In addition to the pain scores on the EHR, satisfaction information will be collected by anesthesia providers conducting the routine post anesthesia care exam 24 hours after delivery. If $10 \%$ reduction in reported pain scores is achieved, the expansion of inclusion criteria for this RPCA program beyond parturients with contraindication to EA is likely; for example, patients with a personal or cultural preference for an IV opioid pain management plan for labor.

\section{Anticipated Outcomes}


The anticipated short term goals for this EBP project can be divided into two groups. The first group pertains to patients focused outcomes and includes: (a) a reduction of $10 \%$ in mean pain score from baseline data, (b) an overall satisfaction rate of $85 \%$ (a $10 \%$ increase from the most recently available HCAPHS data from this hospital on the pain management/childbirth dimension), (c) complication rates within $5 \%$ of baseline rates, and (d) no occurrence of a critical adverse event (Kaiser Permanente Riverside Medical Center, 2018). The second group focuses on the healthcare team and includes: (a) a 90\% rate of completion in remifentanil PCA protocol training, (b) the completion of the order set in the EHR, and (c) a 90\% capture rate of referral for patients receiving prenatal care at this facility who are not eligible for EA.

The anticipated long term goals for this EBP include: (a) expansion of training completion from $90 \%$ to $95 \%$ of staff members, (b) an overall critical adverse occurrence rate of $<1 \%$, and (c) expansion of remifentanil PCA program beyond just those patients not able to receive EA. Ultimately, women who prefer an opioid based plan to EA regardless of reason should be able to access the best and safest option for management of their pain.

\section{Conclusions}

Labor pain is a significant source of anxiety for most pregnant women. Parturients who are excluded from the most common and most effective technique to reduce this pain deserve access to the next best choice. RPCA, due to its fast onset, short half-life and demonstrated effect at reducing pain and boosting satisfaction is the next best option when compared to EA. Adoption of this strategy represents an opportunity to help bring this evidence based technique into a mainstream health system. In time, this technique should improve the labor experience for women at this clinical site and help advance the best practice throughout the United States. 


\section{References}

Aaronson, J., Abramovitz, S., Smiley, R., Tangel, V., \& Landau, R. (2017). A Survey of Intravenous Remifentanil Use for Labor Analgesia at Academic Medical Centers in the United States. Anesthesia and Analgesia, 124(4), 1208-1210.

Anderson, D. (2011). A Review of Systemic Opioids Commonly Used for Labor Pain Relief. Journal of Midwifery \& Women's Health, 56(3), 222-239.

AnimSomuah, M., Smyth, M. R., \& Jones, L. (2011). Epidural versus non-epidural or no analgesia in labour [Systematic Review]. Cochrane Database of Systematic Reviews 2011.

Blair, J. M., Dobson, G. T., Hill, D. A., McCracken, G. R., \& Fee, J. P. H. (2005). Patient controlled analgesia for labour: a comparison of remifentanil with pethidine*. Anaesthesia, 60(1), 22-27.

Centers for Medicare \& Medicaid Services (2017, December 21). HCAHPS: Patients' perspectives of care survey. Retrieved from https://www.cms.gov/Medicare/QualityInitiatives-Patient-Assessment-Instruments/HospitalQualityInits/HospitalHCAHPS.html

Devabhakthuni, S. (2013). Efficacy and safety of remifentanil as an alternative labor analgesic. Clinical Medicine Insights: Women's Health, (6), 37-49.

Douma, M. R., Stienstra, R., Middeldorp, J. M., Arbous, M. S., \& Dahan, A. (2015). Differences in maternal temperature during labour with remifentanil patient-controlled analgesia or epidural analgesia: a randomised controlled trial. International Journal of Obstetric Anesthesia, 24(4), 313-322. 
Douma, M. R., Verwey, R. A., Kam-Endtz, C. E., van der Linden, P. D., \& Stienstra, R. (2010). Obstetric analgesia: a comparison of patient-controlled meperidine, remifentanil, and fentanyl in labour. British Journal of Anaesthesia, 104(2), 209-215.

Evron, S., Glezerman, M., Sadan, O., Boaz, M., \& Ezri, T. (2005). Remifentanil: A novel systemic analgesia for labor pain. Anesthesia and Analgesia, 100(1), 233-238.

Frauenfelder, S., Rijn, R., Radder, C. M., Vries, M. C., Dijksman, L. M., \& Godfried, M. B. (2015). Patient satisfaction between remifentanil patient-controlled analgesia and epidural analgesia for labor pain. Acta Obstetricia et Gynecologica Scandinavica, 94(9), $1014-1021$.

Iowa Model Collaborative. (2017). Iowa model of evidence-based practice: Revisions and validation. Worldviews on Evidence-Based Nursing, 14(3), 175-182.

Kaiser Permanente. (2017, October, 30). Obstetrics pain management guidelines. Unpublished internal document.

Kaiser Permanente Riverside Medical Center. (2018). Labor and Delivery Statistics. Unpublished raw data.

Lee, M., Zhu, F., Moodie, J., Zhang, Z., Cheng, D., \& Martin, J. (2017). Remifentanil as an alternative to epidural analgesia for vaginal delivery: A meta-analysis of randomized trials. Journal of Clinical Anesthesia, 39, 57-63.

Meng, M.-L., \& Smiley, R. (2017). Modern neuraxial anesthesia for labor and delivery. F1000Research, 6 .

Ng, T. K. T., Cheng, B. C. P., Chan, W. S., Lam, K. K., \& Chan, M. T. V. (2011). A doubleblind randomised comparison of intravenous patient-controlled remifentanil with intramuscular pethidine for labour analgesia*. Anaesthesia, 66(9), 796-801. 
Ohashi, Y., Baghirzada, L., Sumikura, H., \& Balki, M. (2016). Remifentanil for labor analgesia: a comprehensive review. Journal of Anesthesia, 30(6), 1020-1030.

Osterman, M. \& Martin, J. (2011, April 6). National Vital Statistics Report: Epidural and spinal use during labor:27-state reporting area, 59(5). Hyattsville, MD: National Center for Health Statistics.

Schnabel, A., Hahn, N., Broscheit, J., Muellenbach, R. M., Rieger, L., Roewer, N., \& Kranke, P. (2012). Remifentanil for labour analgesia: a meta-analysis of randomised controlled trials. European Journal of Anaesthesiology (EJA), 29(4), 177.

Silva, M., \& Halpern, S. H. (2010). Epidural analgesia for labor: Current techniques. Local and Regional Anesthesia, 3, 143-153.

Stourac, P., Suchomelova, H., Stodulkova, M., Huser, M., Krikava, I., Janku, P., Haklova, O., Stoudek, R., Gal, R., \& Sevcik, P. (2014). Comparison of parturient - controlled remifentanil with epidural bupivacain and sufentanil for labour analgesia: randomised controlled trial. Biomedical Papers of the Medical Faculty of the University Palacky, Olomouc, Czechoslovakia, 158(2), 227-232.

Tveit, T. O., Seiler, S., Halvorsen, A., \& Rosland, J. H. (2012). Labour analgesia: a randomised, controlled trial comparing intravenous remifentanil and epidural analgesia with ropivacaine and fentanyl. European Journal of Anaesthesiology (EJA), 29(3), 129-136.

Weibel, S., Jelting, Y., Afshari, A., Pace, L. N., Eberhart, H. L., Jokinen, J., Artmann, T., \& Kranke, P. (2017). Patient-controlled analgesia with remifentanil versus alternative parenteral methods for pain management in labour [Systematic Review]. Cochrane Database of Systematic Reviews 2017. 
Table 1

Distribution of Vaginal Deliveries without Epidural Analgesia

\begin{tabular}{lcc} 
Reason & Number $(\mathrm{n})$ & Percentage \\
\hline Medical Condition & 8 & $5.7 \%$ \\
Natural & 54 & $38.6 \%$ \\
Opioid Plan & 19 & $13.6 \%$ \\
Timing & 59 & $42.1 \%$ \\
Totals & 140 & $100 \%$ \\
\hline
\end{tabular}


Table 2

\begin{tabular}{lcc}
\hline Pain Scores & & \\
& Number (n) & Mean \\
\hline Opioid & 44 & 6.0 \\
No Opioid & 85 & 7.4 \\
\hline
\end{tabular}

Note. The between group difference was significant $(\mathrm{p}=0.0227)$. 